\title{
Balkanologie
}

Balkanologie Revue d'études pluridisciplinaires

Vol. XII, $n^{\circ} 2 \mid 2010$

Volume XII Numéro 2

\section{Dénombrements et recensements de population en Bosnie-Herzégovine durant le XIXeme s. et au début du XXeme s. (I)}

Philippe Gelez

\section{(2) OpenEdition}

Journals

Édition électronique

URL : http://journals.openedition.org/balkanologie/2258

DOI : 10.4000/balkanologie.2258

ISSN : 1965-0582

Éditeur

Association française d'études sur les Balkans (Afebalk)

Référence électronique

Philippe Gelez, «Dénombrements et recensements de population en Bosnie-Herzégovine durant le XIXeme s. et au début du XXeme s. (I)», Balkanologie [En ligne], Vol. XII, n² 2 | 2010, mis en ligne le 07 février 2013, consulté le 17 décembre 2020. URL : http://journals.openedition.org/balkanologie/2258 ; DOI : https://doi.org/10.4000/balkanologie.2258

Ce document a été généré automatiquement le 17 décembre 2020.

(c) Tous droits réservés 


\title{
Dénombrements et recensements de population en Bosnie-Herzégovine durant le XIXeme s. et au début du XXeme s. (I)
}

\author{
Philippe Gelez
}

\section{La collecte}

1 Dans les discussions scientifiques et publiques de l'espace ex-yougoslave, le quantitatif a très tôt acquis une valeur testimoniale et probatoire surestimée au vu des méthodes statistiques qui y ont eu cours jusque dans la seconde moitié du XX ${ }^{\text {ème }}$ siècle. Cette place accordée aux chiffres appelle à un examen critique de la façon dont ils ont été établis et ce qu'ils recouvrent exactement, notamment en Bosnie-Herzégovine - l'un des foyers majeurs d'où jaillissent ces discussions.

2 Faisant suite à une analyse circonstanciée des conversions au XIX ${ }^{\text {ème }}$ siècle en BosnieHerzégovine $^{1}$, le présent article propose donc de consolider les chiffres des recensements humains connus de cette province - ottomane jusqu'en 1878, puis austro-hongroise jusqu'en 1918. Après un examen des méthodes de recensement, on en découvrira les tableaux rectifiés autant que faire se peut, puis une analyse démographique de ces tableaux. À exposer, la méthode que j'ai adoptée et les résultats que je propose demandent beaucoup d'espace; c'est pourquoi mes patients relecteurs de Balkanologie m'ont suggéré de scinder l'article en trois parties. Ici, on trouvera des considérations sur les méthodes de recensement ; dans une seconde partie, je donnerai les chiffres consolidés des recensements. Enfin, l'analyse et l'interprétation de ces chiffres fera l'objet d'un troisième article. Ce qui suit est donc assez factuel et est dépourvu, notamment, de toute dimension comparative avec le reste de l'espace sudslave et balkanique. 


\section{Décomptes ottomans}

3 L'histoire de la statistique moderne en Bosnie-Herzégovine commence, bon an mal an, en 1850. Avant cette date, on ne trouve pour cette province que des registres d'imposition, qu'on appellera ici du nom générique de defter'2.

\section{a. Avant 1850}

4 L'utilisation de ces defter-s par les historiens démographes a fait l'objet de nombreuses remarques méthodologiques, critiques et polémiques ${ }^{3}$. Les deux principales pierres d'achoppement sont l'exhaustivité et la notion de foyer fiscal : 1) l'exhaustivité, car c'est moins l'état «atomique» de la population qui préoccupait la Porte que les ressources qu'elle pouvait en tirer selon un mode d'imposition en majeure partie basé sur la répartition; 2) les foyers fiscaux, car on ignore le plus souvent le rapport numérique qu'ils entretiennent avec les feux réels (feux allumants), dont on ne sait pas non plus par quel nombre moyen il faut les multiplier pour trouver un chiffre approximatif de la population ${ }^{4}$. Ce qu'on va lire plus bas donnera des indications précieuses dans ce sens, mais uniquement pour le XIX ${ }^{\text {eme }}$ siècle. Dans d'autres cas, les registres dénombrent des individus mais seulement dans une certaine catégorie de la population; etc.

5 Ces remarques n'ont pas retenti avec la même force dans toutes les vallées des pays balkaniques. Certains même n'ont presque rien entendu et, dans la lancée des pionniers de la defterologie ${ }^{5}$, continuent d'étudier les registres comme s'ils délivraient un état démographique exhaustif (ou reconstituable avec simplicité) à un moment donné de l'histoire. En Bosnie-Herzégovine, le débat est loin d'être clos, et l'on trouve encore dernièrement des décomptes tout à fait abusifs, qui se donnent pourtant la caution de grands noms. Ainsi l'ottomaniste Ahmed S. Aličić (1934-) a signé une bizarre introduction à l'édition critique d'un defter daté de 1604, dans laquelle il pourfendait d'abord, arguments scientifiques en main, les naïfs qui pensaient trouver un tableau démographique exact en y décomptant les individus; pourtant, à la fin de cette introduction, il procédait à ce décompte ${ }^{6}$. Face à cette situation, les esprits les plus critiques proposent des pistes de réflexion intéressantes mais qui restent rapidement esquissées?.

On donnera un exemple tiré d'un autre ottomaniste, Bruce MacGowan. Les chiffres qu'il trouve dans les registres de capitation (harac) représentent le nombre de chrétiens mâles de plus de 15 ans. Je prends note que l'aire géographique couverte par ces registres n'est pas toujours la même: ceux de 1130/1717-1718 et 1153/1740-1741, notamment, ne comprennent pas l'Herzégovine. On va le voir, cette restriction n'a que peu d'effet sur la validité de ces chiffres. En effet, pour obtenir le nombre de chrétiens, tous âges et sexes compris, MacGowan pense qu'ils doivent être multipliés par trois8 L'historien bosno-herzégovinien Muhamed Hadžijahić (1918-1978) estime quant à lui que le multiplicateur est 3,31 (sans justification), alors même qu'il émet l'hypothèse que la majorité était parfois fixée à dix ans ${ }^{9}-$ le cas de figure le plus probable, d'ailleurs ${ }^{10}$. Enfin, Michael Palairet, un historien britannique spécialiste de l'économie balkanique à l'époque contemporaine, propose un multiplicateur de 3,385 sur la foi de calculs précis pour la Serbie ${ }^{11}$. 
7 Or, la proportion moyenne d'enfants de moins de quinze ans donnée par les recensements austro-hongrois (fin xix ${ }^{\text {eme }}$ siècle), dans une période de paix et dans de meilleures conditions sanitaires, est d'environ $40 \%$ du total des hommes, qu'il faut ensuite multiplier par 0,9 environ pour trouver le nombre de femmes (tous ces chiffres seront justifiés plus bas) - soit un coefficient de 2,66, et non de 3. Pour l'année 1806, j'ai même trouvé que le harac concernait les hommes à partir de sept ans, ce qui réduit encore ce multiplicateur ${ }^{12}$.

8 Qu'on utilise l'un ou l'autre multiplicateur, on trouve facilement une estimation globale dans la mesure où, en Bosnie-Herzégovine, toute la population est imposée au même taux, car un privilège fait que tous appartiennent à la classe la plus basse sur l'échelle d'imposition. Il suffit donc d'une simple multiplication sur la base du chiffre officiel (tableau 1). Malgré tout, les résultats sont tous difficilement acceptables.

Tableau 1. Estimations de population à partir des chiffres du harac

\begin{tabular}{|c|c|c|c|c|c|}
\hline & $\begin{array}{l}\text { Contribuables chrétiens recensés } \\
\text { dans les registres de harac (+ de } \\
\qquad 10 \text { ou } 15 \text { ans) }\end{array}$ & $\begin{array}{l}x 2,66 \\
\text { (Gelez) }\end{array}$ & $\begin{array}{c}x 3 \\
\text { (MacGowan) }\end{array}$ & $\begin{array}{c}\text { x 3,31 } \\
\text { (Hadžijahić) }\end{array}$ & $\begin{array}{c}\text { x 3,385 } \\
\text { (Palairet) }\end{array}$ \\
\hline 1103/1691-92 & 9619 & 25587 & 28857 & 31839 & 32560 \\
\hline 1111/1699-1700 & \multicolumn{5}{|c|}{ Population totalement libérée du paiement du harac } \\
\hline $1112 / 1700-01$ & 12500 & 33268 & 37500 & 41375 & 42313 \\
\hline $1130 / 1717-18$ & 39200 & $\begin{array}{l}104 \\
327\end{array}$ & 117600 & 129752 & 132692 \\
\hline $1145 / 1732-33$ & 43200 & $\begin{array}{l}114 \\
912\end{array}$ & 129600 & 142992 & 146232 \\
\hline $1153 / 1740-41$ & 63440 & $\begin{array}{l}168 \\
839\end{array}$ & 190320 & 209986 & 214744 \\
\hline 1763 & 68545 & $\begin{array}{l}182 \\
330\end{array}$ & 205635 & 226884 & 232025 \\
\hline 1189/1775-76 & 78090 & $\begin{array}{l}207 \\
719\end{array}$ & 234270 & 258478 & 264335 \\
\hline $1202 / 1787-88$ & 98329 & $\begin{array}{l}261 \\
693\end{array}$ & 294987 & 325469 & 332844 \\
\hline 1218/1803-04 & 103327 & $\begin{array}{l}274 \\
850\end{array}$ & 309981 & 342012 & 349762 \\
\hline 1809 & 96000 & $\begin{array}{l}255 \\
360\end{array}$ & 288000 & 317760 & 324960 \\
\hline
\end{tabular}




\begin{tabular}{|c|c|c|c|c|c|}
\hline $1230 / 1814-15$ & 103883 & $\begin{array}{l}276 \\
474\end{array}$ & 311649 & 343853 & 351644 \\
\hline $1235 / 1818 / 1819$ & 98010 & 260 & 294030 & 324413 & 331764 \\
\hline
\end{tabular}

Sources : lignes blanches : MacGowan - en bleu, Hamid Hadžibegić - en orange, Galib Šljivo ${ }^{13}$

Il faut noter qu'avant 1690 , le harac n'est pas un impôt personnel. Je reste volontairement en-dehors du débat sur la sous-déclaration, qui est par nature incontrôlable et donne lieu à des estimations par conséquent injustifiables ; il n'est pas certain que le taux en fût très important ${ }^{14}$. Je me pencherai plutôt ici sur l'évolution de ces chiffres. S'il reste plausible que l'on trouve seulement entre 300 et 350000 chrétiens en Bosnie-Herzégovine en 1818-1819, avec une légère baisse par rapport à quatre années auparavant, c'est parce que le choléra y fait alors des ravages. Mais un octuplement, voire davantage, en l'espace d'un siècle n'est pas envisageable : la petite quarantaine de milliers de 1700-1701 correspond grosso modo au nombre de catholiques (voir plus bas: "Vérifications et compléments»), auxquels il faut adjoindre des orthodoxes de trois à cinq fois plus nombreux au moins ${ }^{15}$.

On a seulement ici le témoignage du perfectionnement des recettes fiscales touchées par la Porte. Des listes de contribuables d'une nouvelle forme du harac, le ianei askeriye, datant de 1858, montrent à l'échelle du sandjak de Travnik que les chiffres qu'on trouve dans ce genre de sources ont une grande souplesse d'acception et peuvent être contradictoires ${ }^{16}$.

11 Du reste, je n'entrerai pas dans le détail de la démographie bosno-herzégovinienne au XVIII ${ }^{\text {eme }}$ siècle : c'est un sujet compliqué, où interviennent guerres et épidémies. Sur la base des quelques réflexions qui précèdent, cependant, j'émets d'assez importantes réserves sur les chiffres donnés par Muhamed Hadžijahić, et que l'on retrouve çà et là ${ }^{17}$. Quoiqu'il en soit, si certains recensements fiscaux, jusqu'au début du XvII ${ }^{\mathrm{e}}$ siècle, ont été plus ou moins compréhensifs de l'ensemble de la population, ce n'est plus le cas au XIX ${ }^{\text {eme }}$ siècle. À cette époque, chaque defter recense une seule catégorie fiscale de la population, au mieux quelques-unes d'entre elles; et ces registres ne sont pas tous composés simultanément. D'autres catégories ne sont jamais recensées. Il est donc impossible d'avoir une série complète, aussi imparfaite fût-elle dans sa méthodologie.

Quelques defter-s annoncent la possibilité d'une étude exhaustive sur un tout petit segment de population: ainsi du registre de garantie mutuelle (kjefilema) de la population de Sarajevo en 1841. On y dénombre (population mâle) 5474 musulmans (68,5\%), 114 Tsiganes (1,4\%), 1660 dhimmi/chrétiens $(20,8 \%)$ et 740 juifs $(9,3 \%)^{18}$, en tout 7988 individus, soit, en y appliquant un coefficient de 0,9 pour trouver le nombre de femmes, env. 15000 habitants. Ce nombre d'habitants est vraisemblable et montre un remarquable développement de la ville de Sarajevo entre 1841 et 1851, comme on le verra plus bas. Il est éloigné des chiffres tout à fait irréalistes que l'on lit souvent dans la littérature jusqu'aux années 1980 (entre 40 et 50000$)^{19}$, ou dans les estimations des observateurs (environ 80000 habitants en $1818^{20}$ ).

Dans l'ensemble, on se contentera d'avoir à l'esprit deux faits marquants pour la période 1800-1850: la révolte serbe de 1804-1815 a probablement amené en Bosnie et en Herzégovine de nombreux musulmans chassés par les Serbes; et la peste de 
1813-1818 a probablement réduit de près du tiers la population provinciale. Les estimations pour cette période varient selon une fourchette très large: des 226000 musulmans et 238000 chrétiens aptes à porter les armes qu'avance le consul autrichien en $1811^{21}$, aux 120000 catholiques, 500000 orthodoxes, 600000 musulmans, 1200 juifs et 30000 Tsiganes que pense trouver le secrétaire du consulat de France en $1808^{22}$. Cette dernière estimation a des reflets de vraisemblance: autour de 1775, les autorités ecclésiastiques de Bosnie-Herzégovine déclaraient environ 70000 catholiques, et il y en aurait eu 200000 en $1830^{23}$. Est-ce le chiffre moyen de 800000 ou 900 000, valable pour 1831, qu'il faut retenir ? ${ }^{24}$ C'est lui que donnent quelques historiens, mais sans le justifier ni citer leurs sources; cette estimation cautionnerait les chiffres élevés du diplomate français, donnés pour la période qui précède la peste de 1813-1818.

\section{b. Déficiences méthodologiques héritées de cette situation dans l'après-1850} uation bosno-herzégovinienne à partir de 1850 sont assez mal connues. Dans l'ensemble, on les qualifiera de dénombrements plutôt que de recensements, selon la distinction établie par les démographes : ils manquent du caractère scientifique que les autorités essayent, dans l'Europe contemporaine, d'attacher à ces instruments d'administration et de décision politique. Leur réalisation n'a pas été confiée à un personnel spécialisé mais aux autorités locales, municipales, ou bien sûr ecclésiastiques; ils suscitent la crainte de la part de la population qui subodore un nouveau tour de vis fiscal, ce qui amène le plus souvent à des sous-déclarations ${ }^{25}$. Jusqu'en 1872-1873 les autorités se contentaient des déclarations des chefs de famille au chef-lieu de district et ne poussaient pas plus loin leurs investigations ${ }^{26}$. De plus, il y a tout lieu de croire que les campagnes de recensement ne furent que très vaguement coordonnées et supervisées, et qu'elles s'étalaient longuement dans le temps (plus de six mois). Ajoutons à cela le fait que la période est troublée: révoltes, opérations militaires, mouvements migratoires, etc. En 1851, deux contrôleurs du recensement furent assassinés par la population du district de Gacko, sur la frontière monténégrine, dans le cadre d'une vengeance qui n'avait cependant pas de rapport avec le recensement lui-même ${ }^{27}$.

\section{c. Nouvelles structures}

Il existait tout de même un semblant d'organisation. À Constantinople, on avait commencé à réfléchir aux méthodes statistiques européennes au moment du 
recensement de 1830-1831, qui n'eut pas lieu en Bosnie en raison d'une grave insurrection. Dans cette province, une deuxième tentative eut lieu entre 1834 et 1836, sous le vizirat de Vecihi Paşa, qui échoua elle aussi ${ }^{28}$. Enfin, une troisième fois, entre 1846 et 1850, sous Tahir Paşa, on lança l'idée d'un recensement de la Bosnie, cette foisci de l'ensemble des contribuables du harac (chrétiens mâles de plus de dix ans). Il semble bien que l'opération, aux mains des popes et des franciscains, se tint, mais on n'en connaît pas les résultats ${ }^{29}$.

17 Ces efforts de réflexion, qui aboutirent, sur le Bosphore, à l'organisation informelle d'un bureau de statistiques en 1868, puis à la création, en 1874, d'une Direction générale des Statistiques en liens étroits avec le Conseil d'État ${ }^{30}$, furent accompagnés de mesures progressives, qui firent sentir leurs effets jusqu'en Bosnie. À Sarajevo fut instaurée en 1853 l'identification numérique des maisons grâce à des petites planches de bois clouées sur les façades ${ }^{31}$; à Mostar, ce fut le cas en $1867^{32}$, année où l'on procéda à une nouvelle numération à Sarajevo, sur de petits ovales de fer-blanc cette fois-ci, avec des chiffres en caractères arabes bien sûr ${ }^{33}$; j'ignore ce qui se fit dans le reste de la province. En 1863, le commissaire impérial Cevdet Efendi (futur Cevdet Paşa, 1822-1885) donna l'ordre de procéder à des dénombrements de population plus fréquents et plus exacts afin de calculer avec justice l'impôt ${ }^{34}$. Par la suite, lorsque la Bosnie fut érigée en vilayet par la loi organique de mai 1865, on instaura un département de statistiques au sein de l'administration du cadastre; à sa tête, un " référent » dont la mission était de contrôler la richesse agricole, foncière et fiscale de la province, et dont les instruments de travail étaient en particulier les dénombrements de population et les livres fonciers. Il avait également la charge de délivrer les passeports et laissez-passer - c'est-à-dire qu'il était censé contrôler les mouvements migratoires : on ne pouvait se déplacer sans avoir justifié le paiement de ses impôts. Il était prévu que le rythme de recensement fût quinquennal, ce qui fut respecté (1870 et 1875) ${ }^{35}$. Jusqu'en 1992 et leur destruction totale, à cause de la guerre, beaucoup de documents émanant de ce département de statistiques se trouvaient à l'Institut oriental de Sarajevo ${ }^{36}$.

18 C'est la date de $1850^{37}$ qui marque ainsi l'apparition de sources exploitables dans un sens statistique, avec de sérieuses réserves cependant. La principale en est que ces dénombrements ne donnent pas d'indications sur la natalité et la mortalité (jusqu'en 1895, d'ailleurs). Entre les différents tableaux de population que nous connaissons, nous ne pouvons que supposer les évolutions, le plus souvent en ayant recours à l'hypothèse d'une progression arithmétique de l'accroissement naturel, et en la pondérant par l'influence qu'ont eue les guerres, les épidémies, les pathologies latentes (telle la syphilis endémique, courante en Bosnie), les migrations, etc.

\section{Recensements austro-hongrois}

19 L'expérience des Austro-hongrois en matière de recensement fait de 1878, a priori, une rupture. Cependant, la mise en place d'une Administration Territoriale vraiment efficiente demanda quelque temps. L'exigence d'autofinancement sur les ressources de la province occupée ne représentait pas le moindre obstacle à l'organisation d'un recensement, opération financièrement très lourde. Par la suite, cependant, structures institutionnelles et moyens allaient être assurés et donner lieu à d'excellentes études. 


\section{a. Recensements austro-hongrois de transition $(1879,1885)^{38}$}

20 réutilisa d'abord les structures existantes, avec leurs procédés de fonctionnement, pour
les faire évoluer par la suite. Aussi les Austro-hongrois considéraient-ils eux-mêmes que le recensement de 1879 n'était pas bon, justement parce qu'il avait été réalisé dans des conditions assez similaires aux précédents : méthodes inquisitoriales aux mains d'un personnel non spécialisé (autorités locales, gendarmes, chefs de village), peur du fisc ou de représailles parmi une population dont une partie, émigrée en Dalmatie, en Slavonie, en Serbie ou au Monténégro durant l'insurrection de 1875-1878, ne semblait pas avoir réintégré son domicile. Enfin, on soupçonna qu'un certain nombre de jeunes hommes avaient passé la frontière par peur de la conscription. Un récit de voyage effectué durant la campagne censitaire semble se faire l'écho des préoccupations populaires en assignant pour but au recensement l'évaluation des forces à disposition pour les chantiers de travaux publics projetés par l'Administration Territoriale (routes notamment) ${ }^{39}$.

e plaçait tout de même dans la lignée des efforts ottomans, qui rencontraient un certain nombre de pratiques auxquelles les hommes de l'Administration Territoriale étaient habitués. On procéda ainsi à la numérotation de toutes les habitations selon une méthode identique à celle de 1853 , avec des planchettes de bois ${ }^{40}$; on recensa les femmes, avec quelques difficultés (surmontées) auprès des musulmans de la région de Banja Luka ; on sépara les étrangers du reste de la population.

Dans l'ensemble, cette campagne de recensement fut réussie vu le peu de moyens à disposition. On parvint notamment à terminer l'opération en un mois. Mais les possibilités informatiques actuelles mettent en lumière de très nombreuses erreurs d'addition, que je détaillerai dans l'article qui suivra celui-ci; de fait, les données, centralisées à Sarajevo, ne furent synthétisées que par un haut fonctionnaire, un statisticien et des pigistes.

Consciente des défauts de l'opération, l'Administration Territoriale déploya de notables efforts pour conférer à ses statistiques la « robustesse » qu'elles avaient dans le reste de la Monarchie. Elle ordonna donc rapidement, en 1885, soit bien avant l'échéance décennale à laquelle l'Autriche était accoutumée, de procéder à un nouveau recensement. La principale modification fut que l'opération fut confiée non plus aux autorités de district, mais à une commission ad hoc sise à Sarajevo, sous la direction d'un haut fonctionnaire, qui avait sous ses ordres 12 contrôleurs, 76 secrétaires et le nombre nécessaire de pigistes et d'employés de bureau. Le travail sur le terrain dura trois mois, et la synthèse 11 semaines.

Malgré ces moyens mis en œuvre, le résultat n'était pas parfait, comme le prouve, encore une fois, le traitement informatique du recensement. Cette fois-ci, cependant, les problèmes sont davantage liés aux ouvriers typographes et au contrôle des épreuves qu'à la synthèse elle-même. En effet, les sous-totaux district par district sont parfois inexacts, mais le total sur l'ensemble de la Bosnie-Herzégovine est, quant à lui, correctement calculé.

\section{b. La réussite des recensements de 1895 et 1910}

L'Administration Territoriale ambitionnait de se doter d'une structure permanente qui aurait la charge de la statistique des provinces occupées. Dès 1840, la Monarchie avait

Balkanologie, Vol. XII, n² 2 | 2010 
mis en place, à Vienne, une Direction de la statistique administrative, transformée en 1863 en Commission centrale de la statistique autrichienne. Karl von Czoernig (1804-1889) dirigea les deux institutions de 1840 à 1865 selon les tendances qui se mettaient en place alors en Europe, avec un accent particulier sur l'ethnographie ; l'une de ses principales réalisations est un livre sur la composition ethnographique de la Monarchie ${ }^{41}$, accompagnée d'une célèbre carte qui parut d'abord en 1846 dans un ouvrage du géographe Heinrich Berghaus (1797-1884) ${ }^{42}$. Quelques mois après le Compromis austro-hongrois de 1867, Budapest ouvrit un bureau statistique exclusivement dédié à la Hongrie ; et dans le lent mouvement d'émancipation qui gagna les autres provinces, des Départements de statistiques furent un peu partout créés, notamment à Czernowicz (Bucovine) en 189143.

C'est au croisement de cette mouvance et des efforts déployés par la Monarchie pour fonder scientifiquement sa politique balkanique qu'il faut replacer la création, le $1^{\mathrm{er}}$ janvier 1894, du Département de statistiques au sein de l'Administration Territoriale de Bosnie-Herzégovine. Le premier directeur en fut Ferdinand Schmid (1862-1925) ${ }^{44}$, plus tard professeur de statistique à l'université d'Innsbruck puis à celle de Leipzig; lui succédèrent Johann Strauss, jusque-là fonctionnaire de l'Administration de Sarajevo, puis Makso Birkovits, d'abord fonctionnaire lui aussi ${ }^{45}$. L'un de ses collaborateurs les plus remarquables fut Karl Maria von Wessely, vice-secrétaire de l'Administration Territoriale ${ }^{46}$.

La conséquence en est que les recensements de 1895 et 1910 sont pour ainsi dire irréprochables d'un point de vue technique. Réalisés par des professionnels, ils contiennent une grande quantité de détails, et un soin minutieux fut apporté à leur publication; d'importantes introductions analysent les données dans leurs grandes lignes, politiques mais aussi scientifiques, de façon claire et précise. Même s'ils ne bénéficièrent pas du traitement mécanique des informations adopté en Autriche dès 1890, les statisticiens du Département rendirent des documents parfaitement cohérents dans leurs calculs.

Par rapport à 1879 et 1885, l'autre grande nouveauté tient au fait que la loi obligea les municipalités (sur la base du découpage fiscal, et non politique, du territoire) à tenir des registres de naissances et de décès à partir du $1^{\text {er }}$ mai $1885^{47}$. Cependant, les résultats du mouvement naturel de la population n'ont été exploités par le Département de statistiques que pour la période 1895-1910: c'est-à-dire que les déclarations ne semblent avoir été centralisée qu'à partir du moment où la loi a obligé les municipalités de les lui adresser, en $1896^{48}$; le Département n'aurait pas récupéré les registres précédents.

\section{Décomptes ecclésiastiques}

29 La période a vu également le développement systématique des décomptes d'âmes chez les catholiques ${ }^{49}$. Introduite officiellement par le Concile de Trente (1545-1563), l'obligation du décompte des baptêmes, mariages et décès a été officialisée en 1585 et probablement appliquée dans la province franciscaine de Bosnie argentine (Bosna Srebrena) avant la fin du $\mathrm{XvI}^{\mathrm{eme}}$ siècle. Beaucoup de registres ont disparu en raison des aléas de l'histoire : guerres (dont l'expédition d'Eugène de Savoie, en 1697), incendies, pillages, etc. ${ }^{50}$ Les plus anciens qu'on a conservés datent de 1641 , et ne sont pas complets. 
30 En revanche, des chiffres permettant de construire des séries régulières pour les catholiques à partir du milieu du XVIII ${ }^{\text {eme }}$ siècle, apparaissent dans les rapports de visitation des vicaires apostoliques de la Province franciscaine de Bosnie argentine. Ces vicaires sont régulièrement issus des rangs des franciscains locaux (seul clergé catholique dans la province) ; ils ont l'obligation de visiter leur territoire de juridiction tous les trois ans ${ }^{51}$. Seul le premier de ces rapports, datant de 1737, est inutilisable car il ne couvre pas l'ensemble des catholiques provinciaux ${ }^{52}$.

31 Puis, à partir de 1836, les franciscains éditent des Stati animarum (" états des âmes ») sous forme d'annuaire, où ils présentent entre autres le nombre et la répartition géographique des fidèles (premiers chiffres en 1855) ${ }^{53}$. Certains historiens y voient un des compléments les plus fiables des recensements, en même temps qu'un excellent moyen de contrôle. Effectués avec une certaine précision, ils permettent de prendre conscience des fausses déclarations et donner des approximations $\mathrm{du}$ nombre d'orthodoxes. Dès les années 1860, ils ont été utilisés dans ce sens; le dernier en date, celui de 1877, a été utilisé par Heinrich Kiepert (1818-1899), le célèbre géographe allemand, dans un essai sur la population de la Bosnie-Herzégovine publié en $1877^{54}$. Un coup d'œil d'ensemble est donné dans le tableau 2 (noter que 1818 est un recensement incomplet).

Tableau 2. Les décomptes des catholiques en Bosnie-Herzégovine entre 1743 et 1877

\begin{tabular}{|c|c|c|}
\hline & Foyers & Âmes \\
\hline 1743 & 4920 & 39942 \\
\hline 1762 & 6812 & 58804 \\
\hline 1768 & 7128 & 60061 \\
\hline 1773 & & 66895 \\
\hline 1776 & 8661 & 73053 \\
\hline 1777 & & 71432 \\
\hline 1779 & 8771 & 76737 \\
\hline 1779 & & 76459 \\
\hline 1786 & 9029 & 68052 \\
\hline 1798 & 11177 & 82422 \\
\hline 1801 & 11946 & 90547 \\
\hline 1806 & 13864 & 98932 \\
\hline 1818 & 8662 & 50928 \\
\hline
\end{tabular}




\begin{tabular}{|l|l|l|}
\hline $\mathbf{1 8 3 7}$ & & 121530 \\
\hline $\mathbf{1 8 4 0}$ & & 128192 \\
\hline $\mathbf{1 8 5 1}$ & & 112000 \\
\hline $\mathbf{1 8 5 5}$ & 17008 & 122581 \\
\hline $\mathbf{1 8 6 4}$ & 18438 & 129271 \\
\hline $\mathbf{1 8 7 7}$ & & 131940 \\
\hline
\end{tabular}

Sources : en bleu, chiffres de Srećko M. Džaja ; en orange, chiffres (peu fiables) de Mladen Lorković, et, en mauve, chiffres de Julijan Jelenić ; en blanc, sources diverses ${ }^{55}$

Je n'ai pas pu vérifier et, le cas échéant, rectifier toutes les lignes. On a pourtant des problèmes de décompte et de méthode. Je prendrai en exemple deux lignes pour lesquelles j'ai pu ébaucher une critique un peu solide.

Les années 1743 et 1768 montrent qu'il faudrait re-consulter les manuscrits. En effet, les chiffres de base sont donnés dans des éditions de source peu critiques, à l'exception de la visitation de 1743 , dont le manuscrit complet a été publié. Une rapide comparaison entre le fac-similé et l'édition indique qu'on ne peut pas faire totalement confiance aux résultats publiés ${ }^{56}$.

L'exemple de 1768 s'avère problématique dans la mesure où l'on possède deux sources qui donnent des chiffres divergents, et que la littérature en tire des résultats encore différents. La première source est la liste par foyers; la seconde en est le résumé. Retravaillée par ordinateur, la première donne un total de 60.452 âmes, alors que le total des sous-totaux inscrits dans la source est de 58.446. La seconde, elle aussi numérisée, donne un chiffre final de 60.256 âmes. Srećko M. Džaja a quant à lui 60.061 âmes. Heureusement minimes, les différences ne devraient cependant pas exister. Pour ma part, je me range au décompte par ordinateur à partir de la source la plus précise.

On rencontre, à peu de choses près, des problèmes identiques avec les stati animarum, que je n'ai pas pu consulter directement: la littérature secondaire se montre discordante, de façon raisonnable cependant. Je prends donc acte des chiffres qu'elle donne, en attendant de pouvoir consulter les sources par moi-même.

En tout état de cause, il faut manier cet indicateur avec circonspection ${ }^{57}$. La méthode de décompte ne correspond aucunement aux exigences des recensements modernes; entre autres, elle n'a pas de date-buttoir, ce qui est gênant dans la mesure où, pour 1743 par exemple, la visite s'est prolongée sur une année, avec un dernier complément bien plus tard: du 15 octobre 1741 au 16 juin $1743^{58}$. Toujours à cette date, d'autre part, les catholiques sont répartis sur trois vicariats (Bosnie, Herzégovine et Raguse). Le vicariat/évêché de Raguse (qui comprend les catholiques des districts de Stolac et Trebinje) n'effectue pas de recensement des âmes en bonne et due forme; on n'en a que des estimations. En fait, seuls les chiffres pour la Bosnie sont à peu près exacts, et ce sont les seuls que je prendrai en compte; mais le vicariat herzégovinien empiète sur la Bosnie civile, si bien que Bosnie franciscaine et Bosnie ottomane ne correspondent pas tout à fait. Parallèlement à ces remarques territoriales, il faut également noter que la comparaison avec les chiffres officiels fait apparaitre des incohérences suspectes. 

ecclésiastique, sur la foi d'un recensement et non des registres de baptême ${ }^{59}$. Cependant, on a la trace d'une estimation interne effectuée par le métropolitain de Sarajevo en 1865 (ce point sera abordé dans l'article suivant).

On n'a strictement rien pour les musulmans, dont les cadres religieux n'ont jamais procédé à un quelconque dénombrement. Enfin, je n'ai pas pu consulter l'ouvrage qui m'aurait permis de savoir s'il avait existé des dénombrements chez les Juifs ${ }^{60}$.

\section{Catégorisation}

La comparaison des catégories ${ }^{61}$ utilisées dans ces divers recensements montre une progressive complexification, principalement assumée par l'administration austrohongroise. Le changement parfois subtil des étiquettes de rubriques ou de colonnes rend difficile, dans certains cas, la comparaison d'une date à l'autre; mais si l'on se contente de réfléchir sur la distribution confessionnelle, qui nous importe ici le plus, on peut synthétiser l'évolution de la façon décrite dans le tableau 3.

Tableau 3. Évolution des catégories de population dans les recensements du XIXème $\mathbf{s .}$

\begin{tabular}{|c|c|c|c|c|c|c|c|c|c|}
\hline 1851 & 1864-1865 & \multicolumn{2}{|c|}{$1869-1870$} & 1875 & 1879 & 1885 & \multicolumn{2}{|l|}{1895} & 1910 \\
\hline Musulmans & Musulmans & \multicolumn{2}{|c|}{ Musulmans } & Musulmans & Mahométans & Mahométans & \multicolumn{2}{|c|}{ Mahométans } & Musulmans \\
\hline \multirow{4}{*}{ Chrétiens } & Grecs & Grecs & Grecs & $\begin{array}{c}\text { Gréco- } \\
\text { orthodoxes }\end{array}$ & $\begin{array}{l}\text { Orientalo- } \\
\text { orthodoxes }\end{array}$ & \multicolumn{2}{|c|}{$\begin{array}{l}\text { Orientalo- } \\
\text { orthodoxes }\end{array}$} & \multicolumn{2}{|c|}{ Serbo-orthodoxes } \\
\hline & Latins & Latins & Latins & s $\begin{array}{c}\text { Rimo- } \\
\text { catholiques }\end{array}$ & $\begin{array}{c}\text { Rimo- } \\
\text { catholiques }\end{array}$ & \multicolumn{2}{|c|}{ Rimo-catholiques } & \multicolumn{2}{|c|}{ Rimo-catholiques } \\
\hline & \multirow[b]{2}{*}{ (Chrétiens) } & & & & & \multicolumn{2}{|c|}{$\begin{array}{l}\text { Protestants } \\
\text { (luthériens, } \\
\text { calvinistes) }\end{array}$} & \multicolumn{2}{|c|}{$\begin{array}{l}\text { Protestants } \\
\text { (luthériens, } \\
\text { calvinistes) }\end{array}$} \\
\hline & & & & & & \multicolumn{3}{|c|}{$\begin{array}{l}\text { Autres (catholiques } \\
\text { arméniens unis, } \\
\text { grecs uniates, } \\
\text { nazaréens, baptistes, } \\
\text { anglicans) }\end{array}$} & $\begin{array}{c}\text { Gréco- } \\
\text { catholiques }\end{array}$ \\
\hline Israélites & Israélites & Israél & ites & Israélites & Israélites & Israélites & $\begin{array}{r}\text { Israélit } \\
\text { (espagn } \\
\text { autres }\end{array}$ & $\begin{array}{l}\text { tes } \\
\text { ols, } \\
\text { s) }\end{array}$ & $\begin{array}{c}\text { Israélites } \\
\text { (séfarades } \\
\text { (espagnols), } \\
\text { autres) }\end{array}$ \\
\hline $\begin{array}{l}\text { Bohémiens } \\
\text { (chrétiens, } \\
\text { musulmans) }\end{array}$ & Bohémiens & Bohém & iens & Bohémiens & & & & & \\
\hline
\end{tabular}




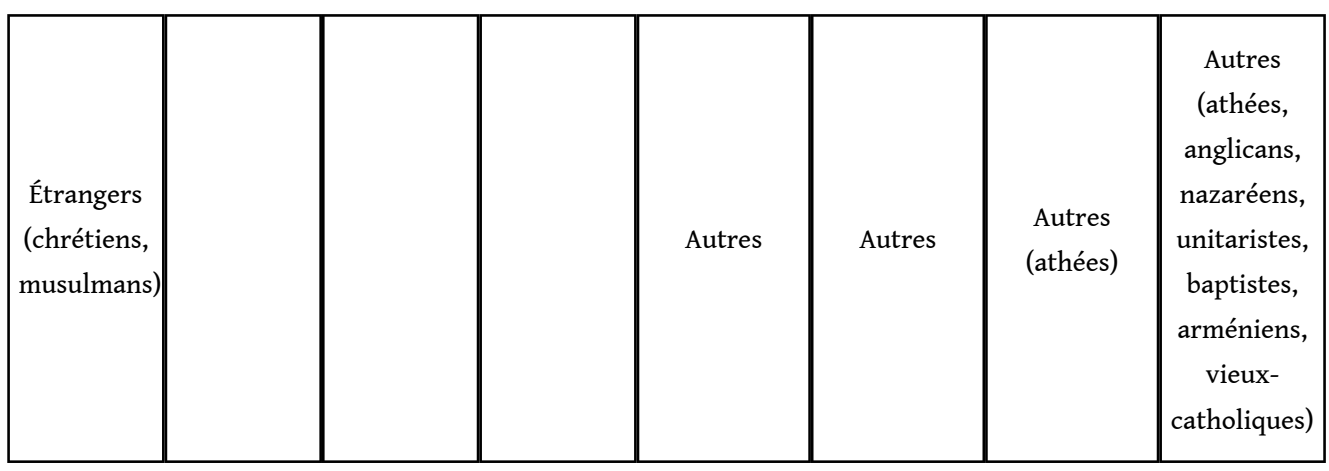

À l'époque ottomane, la catégorisation est exclusivement religieuse (hormis les Bohémiens, sur lesquels je vais revenir); il n'existe pas de considérations sociales (propriétaires, fermiers, etc.), sauf le nombre de personnes par foyer. Les étiquettes « latins » ou " catholiques », d'une part, et « juifs » ou « israélites », d'autre part, sont employés à peu près indifféremment. On trouve également " grecs » et « orthodoxes »: exclusivement le premier dans les documents officiels ottomans, avec une assez nette préférence pour le premier chez les consuls (sauf le russe ?) ou la hiérarchie catholique locale, et jamais dans les documents de provenance serbe ou bosno-orthodoxe. Ce sont le plus souvent les étiquettes référant au centre spirituel, cependant, qui sont utilisées, c'est-à-dire à la définition du groupe selon son millet, et ce sont ces termes que j'ai préférés dans mon tableau. En revanche, les étiquettes religieuses sont soigneusement codifiées à l'époque austro-hongroise.

41 Par ailleurs, le tableau reflète l'ordre dans lequel apparaissent les différentes confessions dans les documents, hormis pour 1910. Hérité de l'époque ottomane où apparaissent les divers groupes en fonction de leur importance politique (musulmans, chrétiens, juifs), cet ordre a été repris par les Austro-hongrois comme une tradition. En 1910, l'ordre se voit modifié : la première confession mentionnée n'est plus l'islam, mais la "serbo-orthodoxie», suivie des musulmans, des catholiques, puis des Juifs, des protestants et, enfin, des « autres ». Ce dernier recensement manifeste l'avènement du droit démocratique que détient le plus fort, quantitativement parlant, à recevoir la première place (les orthodoxes), et les autres à le suivre selon leur importance relative. C'est dans le même sens, quoique dans un contexte historique, politique et rhétorique fort différent, qu'il faut interpréter la seconde distorsion à l'ordre ottoman que j'aie enregistré : dans le Glasnik srpskog učenog društva (Bulletin de la Société savante serbe) est publié en 1866 le tableau récapitulatif de 1864-1865 avec, en tête de chaloupe, les orthodoxes, suivis des catholiques puis des musulmans ${ }^{62}$.

En $1851^{63}$, les catégories sont trompeusement claires. On achoppe en effet sur les Bohémiens (appelés comme tels dans la version française publiée à l'époque) : en Bosnie, ils confessent presque tous l'islam, mais un petit nombre, situé dans le sandjak de Zvornik, est orthodoxe -207 personnes, soit un peu plus de $4 \%$, qui sont des Karavalaques $^{64}$. À ma connaissance, seul le recensement de 1851 établit cette distinction confessionnelle en leur sein. On remarquera que parmi les chrétiens, la séparation entre catholiques et orthodoxes n'est pas effective du point de vue de l'administration. Je connais une seule version de ce recensement doté d'une rubrique "étrangers", divisée entre musulmans et chrétiens ${ }^{65}$. Bref, en tous points ce dénombrement correspond à la catégorisation mise en place pour le recensement de $1830^{66}$. 

dénombrement de population (accompagné d'une enquête agricole), qui cette fois-ci distingue entre orthodoxes et catholiques ${ }^{67}$. On sort en quelque sorte du séculaire faceà-face entre raya et musulman; la pratique administrative se modernise, sans pour autant éclipser le religieux.

Par la suite est instauré un rythme quinquennal : sont effectués deux recensements en 1870 et 1875 . Celui de 1875 est commencé au début de l'année, mais les circonstances de la guerre dont la Bosnie est l'un des théâtres en empêchent la réalisation correcte. On en présentera tout de même les chiffres.

Le recensement austro-hongrois de 1879 se caractérise par une structure légèrement modifiée en raison des pratiques austro-hongroises. Pour les orthodoxes, il double l'habituelle étiquette "grecque " d'un "orthodoxe»; les bohémiens n'apparaissent plus, conformément à l'usage autrichien qui ne les prend pas en compte en tant que tels. Les musulmans, par habitude et selon l'usage encore une fois, sont appelés «mahométans» («Mohamedaner» en 1879 et 1885, « Muhamedaner» en 1895). Les femmes sont désormais dénombrées et ce, distinctement des hommes. La principale innovation réside dans la catégorie religieuse appelée " autre ", où sont regroupés tous ceux qui n'appartiennent pas aux quatre confessions principales. Cette extension de la population statistique parachève de généraliser la portée administrative du recensement qui, perdant de sa valeur proprement fiscale, tente de donner un tableau démographique et économique, et de servir plus directement de source à l'action politique. De l'aveu même de l'Administration Territoriale, c'était son seul but.

Cette nouvelle dimension gagne toujours plus en visibilité par la suite. En 1885, le procédé reste le même; mais cette fois-ci, on publie également une division de la population selon la situation maritale ainsi que selon l'activité professionnelle, et on précise le nombre d'étrangers. Le statut religieux se juridicise: les catholiques sont appelés « rimo-catholiques », les orthodoxes « gréco-orthodoxes ». En 1895 et 1910, on procède à un recensement extrêmement complet et précis de la population. Outre de nouvelles données sur la population militaire et étrangère, les catégorisations restent stables (religion, statut marital, profession), mais elles se fragmentent : pour la religion, qui demeure la seule division ethnique, on crée en 1895 une catégorie " chrétiens » où sont distingués gréco-orthodoxes, rimo-catholiques, protestants et «autres ». Notre tableau donne le détail de ce qui y est enregistré ; tous ces nouveaux groupes religieux sont apparus avec l'immigration en provenance des autres provinces habsbourgeoises, et représentent, numériquement, des micro-minorités. En 1910, la catégorie englobante des "chrétiens" disparaît au profit d'une individualisation de chaque confession chrétienne: les serbo-orthodoxes (appellation officielle à partir de 1906) sont totalement séparés des catholiques, qui se trouvent quant à eux divisés entre rimo- et gréco-catholiques; les protestants forment une colonne à part, et les autres sectes chrétiennes sont remisée dans une large catégorie "autres». Les musulmans sont désormais désignés comme tels, et non plus comme mahométans, suite à une polémique lancée en 1899.

47 L'ensemble de cette évolution montre une complexification catégorielle remarquable, due aux progrès techniques introduits par la Monarchie aussi bien qu'à l'évolution démographique elle-même. À l'époque ottomane, cette évolution montre la lente adoption des standards européens. À l'époque austro-hongroise, elle trahit que l'administration de Sarajevo a voulu transférer le potentiel politique des communautés

Balkanologie, Vol. XII, nº 2 | 2010 
religieuses entre ses mains, en tentant de réduire l'identité religieuse à sa simple motivation spirituelle; le recensement de 1910 entérine cependant une sorte d'échec, puisqu'il restaure les anciennes divisions tout en les réarrangeant dans la perspective démocratique. La catégorisation a échoué à maîtriser la population. On se trouve à la croisée de la tradition de recensement autrichienne/austro-hongroise, où les groupes religieux apparaissent depuis la moitié du XviII ${ }^{\text {eme }}$ siècle (1751), et de la politique de Béni von Kállay (1839-1903), le ministre commun des finances en charge de l'administration de la Bosnie-Herzégovine entre 1882 et 1903, qui désirait dégager un consensus national fort dans la province afin d'en transcender les clivages politiques fondés sur la religion.

Exactement identique par sa structure aux campagnes de recensement qui furent simultanément menées dans la Monarchie, le tableau statistique de 1910 ajoute encore un certain nombre de distinctions (sexes, âges) et d'informations (illettrisme, langues parlées, etc.). La plus notable relève la langue maternelle des recensés : on appliquait enfin à la Bosnie-Herzégovine le principe linguistique dans la définition des groupes, principe qui avait fait couler beaucoup d'encre dans le reste de la Monarchie depuis le Congrès démographique international tenu à Vienne en 185768. Ces diverses informations ne furent publiées que sous forme de tableau sommaire (au niveau du district), et non pour chaque localité.

Les catégorisations appliquées dans les recensements de la Bosnie-Herzégovine depuis 1850 n'ont guère contribué à formaliser les nationalismes; elles ont plutôt essayé d'en retarder la maturation, et ont contribué à ce qu'ils se construisent sur une base exclusivement religieuse. Les processus en cours dans la province à partir de 1879 ne sont donc pas assimilables à ce qui se passe dans le reste de Monarchie ${ }^{69}$.

\section{Conclusion}

L'examen de ces méthodes de comptage des âmes montre un réel progrès technique à partir des années 1850 , et qui prend ses racines au début du XVIII ${ }^{\text {ème }}$ siècle. Il n'en reste pas mois que l'année 1878, date de l'entrée de l'administration austro-hongroise en Bosnie-Herzégovine, continue à signifier une rupture : technique et esprit systématique vont désormais s'emparer du corps populaire et le mesurer, le jauger, l'individualiser définitivement, et faire correspondre la réalité humaine à une réalité exactement chiffrée. La seconde partie de cet article s'intéressera à vérifier ces chiffres.

1.

2.

\section{NOTES}

Gelez (Philippe), « Se convertir en Bosnie-Herzégovine (c. 1800-1918) », Süd-Ost Forschungen, 68, 2009. 
Pour une bibliographie détaillée de tout ce qui existe sur la Bosnie-Herzégovine, voir Kupusović (Amina), "Tâpû Tahrîr Defteris Relating to Bosnia», in Koller (Markus), Karpat (Kemal H.), Ottoman Bosnia. A History in Peril, Madison : The University of Wisconsin Press / Kemal H. Karpat, 2004 ; et, en complément, Gelez (Philippe), Petit guide pour servir à l'étude de l'islamisation en Bosnie et en Herzégovine, avec le recueil des sources connues, ainsi qu'un commentaire sur l'utilisation qui a été faite de celles-ci, Istanbul : ISIS, 2005, pp. 133-141.

3. De façon synthétique et rapide, consulter Eldem (Vedat), ed., The Population of the Ottoman Empire and Turkey, 1500-1927, Ankara: State Institute of Statistics, 2003 [Première édition : Cem Behar, 1996]; et Behar (Cem), «Qui compte?», Histoire \& Mesure, 18 (1-2), 1998. À ma connaissance, les premières remarques méthodologiques ont été exposées de façon systématique par Fine (John V. A.), The Bosnian Church : A New Interpretation. A Study of the Bosnian Church and Its Place in State and Society from the 13th to the 15th Centuries, New York/ London: Columbia University Press, 1975, pp. 79-80. Ce n'est qu'une dizaine d'années ensuite que ces critiques se sont enracinées chez les historiens. Voir notamment Lowry (Heath W. Jr.), « The Ottoman Tahrîr Defterleri as a Source for Social and Economic History : Pitfalls and Limitations ", texte donné lors d'une conférence en 1986 (consulté dans Studies in Defterology. Ottoman Society in the Fifteenth and Sixteenth Centuries, Istanbul: ISIS, 1992; Beldiceanu (Nicoară), Beldiceanu-Steinherr (Irène), «Les recensements ottomans effectués en 1477, 1519 et 1533 dans les provinces de Zvornik et Hercégovine ", Turcica, 22, 1988 ; Murphey (Rhoads), "Ottoman Census Methods in the MidSixteenth Century. Three Case Histories", Studia Islamica, 1990; Ataman (Bekir Kemal), "Ottoman Demographic History (14th-17th Centuries). Some Considerations", Journal of the Economic and Social History of the Orient, 25, 1992; Ursinus (Mickael), Quellen zur Geschichte des osmanischen Reiches und ihre Interpretation, Istanbul: Isis, 1994. Voir aussi Todorov (Nicolai), Velkov (Asparuh), éds., Situation démographique de la péninsule balkanique(fin du Xve s. - début du XVI s.), Sofia : Académie bulgare des sciences, 1988.

4. Les defter-s enregistrent les foyers, les veuves et les célibataires; pour les foyers, par quel coefficient multipliera-t-on les chiffres obtenus? Barkan proposait le coefficient 5 dans son article de 1958 en en soulignant l'arbitraire dans certains cas; il parlait de "présomption " (Barkan (Ömer Lutfi), «Essai sur les données statistiques des registres de recensement dans l'Empire ottoman aux XV et XVI ${ }^{\mathrm{e}}$ siècles ", Journal of the Economic and Social History of the Orient, 1, 1958, p. 28). Ce coefficient a fait l'objet de multiples débats, en particulier parce qu'il semblait établi de façon arbitraire.

5. Barkan (Ömer Lutfi), art.cit. ; Cvetkova (Bistra), « Les tahrir defterleri comme sources pour l'histoire de la Bulgarie et des pays balkaniques ", Revue des Études sud-est européennes, 16 (1), 1978. 6. Aličić (Ahmed S.), «Uvod ", in Handžić (Adem), ur., Opširni popis bosanskog sandžaka iz 1604. godine (Recensement détaillé du sandjak de Bosnie de 1604), Sarajevo: Bošnjački institut / Orijentalni institut, 2000, sv. I/1, pp. iii-xxxvii (en anglais pp. xli-li, où n'apparaissent que les décomptes abusifs). Voir aussi la confiance aveugle qu'accorde Muhamed Hadžijahić au register de captiatio n(harac): ХАџИЈАХИЋ (МУХАМЕД), «НЕКИ РЕЗУЛТАТИ ИСПИТИВАЊА ЕТНИЧИХ КРЕТАҢА У БОСНИ У XVIII И ПРВОЈ ПОЛОВИЦИ XIX СТОљЕЋА » (Quelques résultats de l'étude des migrations ethniques en Bosnie-Herzégovine durant la première moitié du XIX ${ }^{\text {ème }}$ siècle), Prilozi Instituta za istoriju, 11-12, 1975-1976, pp. 289-300, et surtout ХАџИЈАХИЋ (МУХАМЕД), «МЕТОДОЛОШИ ПОСТУПЦИ КОД УТВРЪИВАњА БРОЈА БОСАНСКЕ ПОПУЛАЦИЈЕ У КАСНИЈЕМ ТУРСКОМ ПЕРИОДУ » (Méthodologie pour consolider les chiffres de la population bosniaque à la fin de la période turque), Prilozi Instituta za istoriju, 11-12, 1975-1976, pp. 300-301.

7. Đurđev (Branislav), «Neke napomene o islamizaciji i bošnjaštvu u istoriji Bosne i Hercegovine " (Quelques remarques sur l'islamisation et la nation bochniaque dans l'histoire de la Bosnie-Herzégovine), Prilozi za orijentalnu filologiju, 41, 1991, pp. 27-28 ; Moačanin (Nenad), 
«Bosansko-humski krstjani u turskim vrelima (napomene)», in Šanjek (Franjo), ur., Fenomen "Krstjani" u srednjovjekovnoj Bosni i Humu. Zbornik radova (Le phénomène des "Chrétiens" en Bosnie et Hum médiévaux), Sarajevo / Zagreb : Institut za istoriju / Hrvatski institut za povijest, 2005, pp. 407-412 (notamment la note 1 p. 407).

8. MacGowan (Bruce), Economic Life in Ottoman Europe. Taxation, trade and struggle for land, 1600-1800, Cambridge / Paris : Cambridge University Press / MSH, 1981, p. 96. 1112 : avec Hersek et Izvornik; 1130 : sans Hersek, avec Izvornik; 1153 : sans Hersek, avec Izvornik; 1202 : avec Hersek et liva-i Seri Selim (?) ; 1230 : avec Hersek et liva-i Seri Selim (?). À noter que MacGowan ne cherchait pas à obtenir de chiffres précis, mais uniquement une tendance démographique sur le long terme. Džaja reprend ces chiffres sans souligner leur disparité géographique (Džaja (Srećko M.), Konfesionalnost i nacionalnost Bosne i Hercegovine. Predemancipacijsko razdoblje 1463.-1804,Mostar : ZIRAL, 1999, p. 102).

9. ХАШИЈАХИЋ (МУХАМЕД), « НЕКИ РЕЗУЛТАТИ » (art.cit.), pp. 289-290.

10. ФИЛИПОВИЋ (МИЛЕНКО С.), « ПОПИС СРБА ХАРАЧКИХ ОБВЕЗНИКА У МОДРИЧИ И ОКОЛИНИ 1851 ГОДИНЕ » (Liste de harac pour Modriča et ses environs en 1851), РАДОВИ НД НРБИХ, Х (4), 1958, pp.114, 123.

11. Palairet (Michael), The Balkan Economies c. 1800-1914. Evolution without Development, Cambridge : Cambridge University Press, 1997, p. 5.

12. Šamić (Midhat), «Francuski izvještaji o Bosni početkom XIX vijeka (1806-1813)» (Rapports d'agents français sur la Bosnie au début du XIX ${ }^{\text {ème }}$ siècle), Građa, XI (8), 1961, p. 15.

13. MacGowan (Bruce), op.cit. ;Hadžibegić (Hamid), Glavarina u Osmanskoj državi, Sarajevo : OIS, 1966, pp. 93, 102, 103, 106, 108-109 (chiffres pour l'eyalet de Bosnie, bien plus étendu que la province austro-hongroise); Šljivo (Galib), Bosna i Hercegovina 1813-1826 (La Bosnie-Herzégovine 1813-1826), Banjaluka : Institut za istoriju, 1985, pp. 28-30 (chiffres pour tout l'eyalet de Bosnie).

14. Milenko Filipović pense qu'il n'y avait pas de sous-déclaration dans les listes de 1851, à une époque où, il est vrai, la collecte de cet impôt avait été réformée et où la possibilité d'y échapper s'était considérablement amoindrie (Филиповић (милЕнко с.), art.cit., pp.113-116).

15. Ramiza Smajić, chercheur de l'Institut d'histoire de Sarajevo, est en train de travailler sur la démographie de la Bosnie-Herzégovine au tournant du XVIII ${ }^{\mathrm{eme}}$ siècle.

16. Hadžibegić (Hamid), op.cit., p. 138-139.

17. Par exemple, Džaja (Srećko M.), op.cit., p. 81.

18. Mestvica (Mula Muhamed), Popis uzajamnog jamčenja stanovništva u Sarajevu iz 1841. godine. (Liste de caution solidaire de la population de Sarajevo en 1841), Sarajevo : Muzej grada Sarajeva, 1970. Avdo Sućeska, auteur de l'introduction à l'édition de ce defter, n'a pas indiqué les totaux; ceux-ci sont donnés par Hana Younis (Hana (Younis), «Skice porodičnog života u Sarajevu posljednjih decenija osmanske vladavine " (Esquisse de la vie familiale à Sarajevo durant les dernières décennies de la domination ottomane), Prilozi Insituta za istoriju, 36, 2007, pp. 35-36. Mes propres calculs divergent imperceptiblement des siens.

19. Il est étonnant que Michael Palairet les reprenne (Palairet (Michael), op.cit., p. 31). Pour une remarque critique sur ces chiffres, voir ХАџИЈАХИЋ (МУХАМЕД), « МЕТОДОЛОШИ ПОСТУПЦИ » (art.cit.), p. 300.

20. Šljivo (Galib), op.cit., pp. 135-136.

21. Kapidžić (Hamdija), ur., Prilozi za istoriju Bosne i Hercegovine u XIX vijeku (Contributions à l'histoire de la Bosnie-Herzégovine au XIX ${ }^{\text {eme }}$ siècle), Sarajevo : Naučno društvo NR BiH, 1956, p. 9 note 7 ; ХАџИЈАХИЋ (МУХАМЕД), « НЕКИ РЕЗУЛТАТИ » (art.cit.), p. 291.

22. Cité d'après ПЕЈАНОВИЋ (ЂоРТЕ), СТАНОВНИШТВО БОСНЕ И ХЕРЦЕГОВИНЕ [La population de la Bosnie-Herzégovine], Beograd : Naučna knjiga, 1955, p. 28.

23. Baltić (Jako), Godišnjak od događaja crkvenih, svjetskih i promine vrimena u Bosni [Chronique des événements ecclésiaux et mondains, ainsi que des variations météorologiques en Bosnie], Sarajevo : Veselin Masleša, 1991, p. 45 (1773 : 8412 foyers et 66895 âmes catholiques en Bosnie), 
51 (1777 : 8629 foyers et 71442 âmes catholiques en Bosnie) et 83 (1830: 200000 env. catholiques en Bosnie et en Herzégovine). Nous aurons l'occasion de revenir sur les décomptes franciscains.

24. MacGowan (Bruce), op.cit., p. 202.

25. Dupâquier (Jacques), Dupâquier (Michel), Histoire de la démographie. La statistique de la population des origines à 1914, Paris : Perrin, 1985, p. 288 ; Dupâquier (Michel), Démographie, Paris : PUF, 2001, p. 15.

26. CADN (Centre des Archives Diplomatiques de Nantes), Poste consulaire de Bosna-Seraï/ Sarajevo (Sarajevo), vol. 3, 15.IV.1872 et 17.IX.1873.

27. ШИШИЋ (ФЕРДО), ur., БОСНА И ХЕРЦЕГОВИНА ЗА ВЕЗИРОВАҢА ОМЕР-ПАШЕ ЛАТАСА (1850-1852). ИСПРАВЕ ИЗ БЕЧКОГ ДРЖ. АРХИВА (La Bosnie et l'Herzégovine sous le vizirat d'Ömer Paşa Latas (1850-1852). Documents des Archives d'État de Vienne), Subotica: SKA, 1938, p. 408.

28. ХАџИЈАХИЋ (МУХАМЕД), « НЕКИ РЕЗУЛТАТИ » (art.cit.), p. 290.

29. ПЕЈАНОВИЋ (ЂОРЪЕ), ор.cit., p. 28.

30. Karpat (Kemal H.), "The Ottoman Adoption of Statistics from the West in the $19^{\text {th }}$ Century ", in Karpat (Kemal H.), Studies on Ottoman Social and Political History. Selected Articles and Essays, Leyden : Brill, 2002, p. 134.

31. On surnomma «Tahtar» ou « Mühendis» Mehmed Kiamil Pacha, le commissaire spécial qui était à l'origine de cette initiative (il avait été vizir de la province en 1845 - voir Hadžihuseinović (Salih Sidki, Muvekkit), Povijest Bosne. S turskog preveli Abdulah Polimac et al., Sarajevo : El-Kalem, 1999, vol. II, pp. 1005, 1063. À noter qu'auparavant, les maisons portaient peut-être un numéro, mais que celui-ci ne semble pas avoir été alloué de façon durable (Mestvica (Mula Muhamed), op.cit., passim) ; c'est une éventualité que soulève Milenko Filipović, pour l'écarter aussitôt, à propos des environs de Modriča en 1851 (Филиповић (милЕнко с.), art.cit., pp. 112-113.

32. Hasandedić (Hivzija), Spomenici kulture turskog doba u Mostaru (Monuments culturels de l'époque turque à Mostar), Sarajevo : Veselin Masleša, 1980, p. 8.

33. Ljiljak (Milan), Pošta, telegraf i telefon u Bosni i Hercegovini. I. Razvoj i razmještaj mreže poštanskog i telegrafskog saobraćaja za vrijeme osmanlijske vlade, Sarajevo : Preduzeće PTT Saobraćaja, 1975, p. 108.

34. CADN, Poste vice-consulaire de Mostar, vol. 6, 18 et 24.XII.1863.

35. CADN Sarajevo, vol. 4, 15.VII.1874 ; vol. 5, 16.X.1875.

36. Aličić (Ahmet S.), Uređenje bosanskog ejaleta od 1789. do 1878. godine (L'organisation de l'eyalet de bosnie entre 1789 et 1878), Sarajevo: Orijentalni institut, 1983, pp. 101-102, 118 ; Dursun (Selçuk), Population Policies of the Ottoman State in the Tanzimat Era 1840-1870, manuscrit de MA de la Sabanci University, 2001, pp. 26-27 (disponible sur internet: digital.sabanciuniv.edu/tezler/ tezler/ssbf/master/dursuns/toc.pdf). Voir aussi Karpat (Kemal H.), art.cit., p. 137.

37. Kemal Karpat mentionne un recensement général de la population mâle de BosnieHerzégovine pour 1844 ; je n'en ai trouvé trace nulle part. D'après lui, il n'y aurait pas eu d'autres recensements jusqu'en 1877, et celui-ci n'aurait été qu'une mise à jour de celui de 1844. Il ignore donc l'existence de quatre autres recensements $(1851,1865-1866,1870,1875)$, et le fait que celui de 1877 est une mise à jour de celui de 1875, voire de 1870 (Karpat (Kemal H.), Ottoman Population 1830-1914. Demographic and Social Characteristics, London : University of Wisconsin Press, 1985, p. 121).

38. Pour cette sous-partie, voir Hauptresultate der Volkszählung in Bosnien und der Hercegovina vom 22. April 1895, nebst Angaben über territoriale Entheilung, öffentliche Anstalten und Mineralquellen. Zusammengestellt vom Statistischen Departement der Landesregierung, Sarajevo: LandesdruckereiZemaljska štamparija, 1896, pp. III-VIII.

39. Zirdum (Andrija), « Bosna u ljeto 1879. godine. Uz 160. obljetnicu rođenja putopisca Đure Pilara » (La Bosnie en 1879. À l'occasion du $160^{\mathrm{eme}}$ anniversaire de la naissance de l'écrivain Đuro Pilar), Bosna Franciscana 25 (2006), p. 285. 
40. C'est ce que l'on constate dans l'ordonnance indiquant aux recenseurs les démarches à suivre pour le recensement de 1885 (Sammlung der für Bosnien und die Hercegovina erlassenen Gesetze, Verordnungen und Normalweisungen 1885, pp. 50-76).

41. Czoernig (Karl von), Ethnographie der oesterreichischen Monarchie. Mit einer ethnographischen Karte in vier Blaettern, Vienne : Hof- und Staatsdruckerei, 1855-1857, 4 vols.

42. "Ethnographische Karte der Osterreichischen Monarchie. Nach Bernhardi, Safarik, und eigenen Untersuchungen von HBgs. Febr 1845. », in Berghaus (Heinrich), Physikalischer Atlas oder Sammlung von Karten, auf denen die hauptsachlichsten Erscheinungen der anorganischen und organischen Natur nach ihrer geographischen Verbreitung und Vertheilung bildlich dargestellt sind, vol. II, Gotha : Justus Perthes, 1848. Sur les usages politiques de cette carte, voirLabbé (Morgane), "Les usages diplomatiques des cartes ethnographiques de l'Europe centrale et orientale au XIX ${ }^{\mathrm{e}}$ siècle », Genèses, (68), 2007 (dossier « Gouverner par les cartes »). Czoernig est également l'auteur d'une monumentale ethnographie (voir note précédente).

43. Meyer (Maximilian), «Discours en honneur du jubilée cinquanténaire de la Commission Centrale de Statistique autrichienne », Bulletin de l'Institut international de statistique, 20 (1), 1915 ; Juraschek (Franz von), « Bericht über die Fortschritte der Statistik in Oesterreich seit 1891, für die IV. Session des internationalen statistischen Institutes ", Bulletin de l'Institut international de statistique, 8 (1), 1895 ; Ficker (Adolph), « Skizze einer Geschichte des k. k. statistischen Bureau's in den Jahren 1829 bis 1866 ", inStatistisch-administrative Vorträge auf Veranstaltung der k.k. statistischen Central-Commission abgehalten im Winter-Semester 1866-1867., Wien: Hof- und Staatsdruckerei, 1867. Sur l'origine de la statistique hongorise, on peut consulter Horváth (Róbert A.), «Étienne Hatvani et les origines de l'arithmétique politique en Hongrie », Population, 14 (4), oct.-déc. 1959. Sur l'ensemble de la question, consulter Horváth (Róbert A.), Le Développement de l'École de Statistique descriptive allemande. Une synthèse de l'histoire scientifique en statistique, Szeged : Szegedi nyomda, 1981 ; Dupâquier (Jacques), Dupâquier (Michel), op.cit., pp. 270-271.

44. Ferdinand Schmid était auparavant fonctionnaire aulique (Hofconcipist) de la Commission centrale de statistiques de Vienne (voir « Mittheilungen und Miscelen ", Statistische Monatschrift, 20,1894 , pp. 13, 77). Après son départ, causé par sa nomination en tant que professeur à l'Université de Leipzig, Schmid resta en liens étroits avec la Bosnie-Herzégovine, écrivit une monographie encore incontournable sur l'administration austro-hongroise où il synthétisa un grand nombre de statistiques qu'il avait lui-même contribué à établir ; pendant la guerre, il continua à s'intéresser de près à l'histoire économique de la région (cf. Kapidžić (Hamdija), ur., Naučne ustanove u Bosni i Hercegovini za vrijeme austrougarske uprave (Institutions scientifiques en Bosnie-Herzégovine durant l'administration austro-hongroise), Sarajevo: Arhiv Bosne i Hercegovine, 1973, doc 240, pp. 519-520 ; Deutsche Biographische Enzyklopädie, München, Saur, 1995-2000, vol. 8, p. 701).

45. Rezultati popisa žiteljstva u Bosni i Hercegovini od 10. oktobra 1910, sastavio Statistički odsjek Zemaljske vlade sa preglednom kartom konfesija, Sarajevo : Zemaljska štamparija, 1912, p. V ; Hunski (Vjekoslava), «Naučno-izdavačka djelatnost u Bosni i Hercegovini u okviru Statističkog departmana Zemaljske vlade, Meteorološke stanice i Geološkog zavoda (1878-1918) » (L'édition scientifique en Bosnie-Herzégovine dans le cadre du Département statistique de l'Administration territoriale, du Service météorologique et de l'Institut géologique), Prilozi Instituta za istoriju, 19, 1982, pp. 291-292. On trouve chez Tomislav Kraljačić, que le Département fut fondé en 1896 (Kraljačić (Tomislav), Kalajev režim u Bosni i Hercegovini (1882-1903) [Le régime de Kállay en Bosnie-Herzégovine (1882-1903)], Sarajevo : Veselin Masleša, 1987, p. 436) ; ce qui est troublant, c'est que Kraljačić reprend son information de Ferdinand Schmid lui-même (Schmid (Ferdinand), Bosnien und die Herzegowina unter der Verwaltung Österreich-Ungarns, Leipzig: Veit, 1914, p. 191), que je n'ai pas eu l'occasion d'aller reconsulter pour cet article. 
46. À défaut de consulter les personalia des fonctionnaires du Département de statistique, on peut en suivre la carrière dans le Bosnischer Bote / Bosansko-hercegovački glasnik (par ex. 3, 1899, p. 94 et 4, 1900, p. 88).

47. Gesetze und Verordnungen 1885, pp. 84-96, 343-344 (circulaire du 6.VI.1885).

48. ABH (Archives de Bosnie-Herzégovine), fonds de l'Administration Territoriale à Sarajevo (ZVS), 14 15-3/1904.

49. Il aurait fallu que je consulte un ouvrage de référence auquel je n'ai malheureusement pas eu accès pour la rédaction de ce travail : Draganović (Krunoslav), Opći šematizam katoličke crkve u Jugoslaviji, Sarajevo : Akademija Regina Apostolorum, 1939.

50. Jukić (Milo), «Popis umrlih od kuge u župi Kreševo 1765. godine » (Liste des morts de la peste dans la paroisse de Kreševo de 1765), Bosna Franciscana, 25, 2006, p. 305 jerković (Mijat), Hrvati plehanskog kraja sredinom XVIII. stoljeća (1742.-1771.). Najstarije matice župe Velika (1763.-1771.) i popisi katolika iz 1742. $i$ 1768. (Les Croates du pays de Plehan au milieu du $18^{\text {eme }}$ siècle. Les plus anciens registres de la paroisse de Velika (1763-1771) et les dénombrements de catholiques de 1742 et 1768), Sarajevo / Plehan: Svjetlo riječi / Slovoznak, 2006, pp. 67-72 (les archives du monastère de Kreševo ont brûle le 7 avril 1765). Stipetić et Vekarić sont mal renseignés sur le sujet (Stipetić (Vladimir), Vekarić (Nenad), Povijesna demografija Hrvatske (Démographie historique de la Croatie), Zagreb / Dubrovnik : HAZU, 2004, p. 18).

51. Sur la pratique des visites pastorales (canoniques), par l'évêque, qui doit se faire chaque année, voir Coulet (Noël), Les visites pastorales, Turnhout : Brepols, 1977.

52. Đaković (Luka), ur., Prilozi za demografsku i onomastičku građu Bosne i Hercegovine [I] (na osnovu popisa katoličkog stanovništva 1743. godine) [Matériaux démographiques et onomastiques bosnoherzégoviniens (recensement de la population catholique de 1743], Sarajevo : ANUBiH, 1979, p. 12 ; Džaja (Srećko M.), op.cit., p. 149.

53. ХАџИЈАХИЋ (МУХАМЕД), « НЕКИ РЕЗУЛТАТИ » (art.cit.), pp. 289, 300.

54. Kipert (Hienrich), "Anhang", in Blau (Otto), Reisen in Bosnien und der Herzegowina. Topographische und pflanzengeographische Aufzeichnungen, Berlin : Dietrich Reimer, 1877.

55. Džaja (Srećko M.), op.cit., p. 150 ; Chiffres en orange et mauve consultés chez Pejanović (l'exemple des années 1743 et 1779 montre que ces calculs sont peu fiables et devraient être repris. ПЕЈАНОВИЋ (ЋОРъЕ), op.cit., p. $26 ; 1855$ : le Schematismus de 1856 indique un total de 122 865, alors que le calcul mécanique à partir des paroisses donne 122581 (Source : MAE Paris, Correspondance consulaire et commerciale (CCC), Bosna-Seraï vol. 1, 21.II.1863. - 1864 : MAE CCC ibid., 8.XII.1867. - 1877 : calculé à partir du détail des localités donné par Kiepert (Kipert (Hienrich), art.cit.). Ont également été publiés les chiffres de la visitation de 1818, effectuée par l'évêque Miletić (Šljivo (Galib), op.cit., n. 7 p. 142). Cette visitation, qui ne couvrait pas l'ensemble de la Bosnie, a compté 8662 foyers pour 50928 âmes.

56. Mandić (Dominik), ed., Chroati catholici Bosnae et Hercegovinae in descriptionibus annis 1743 et 1768 exaratis. Iuxta codices autographos qui in Archivo S. C. de Propagada Fide exstant edidit P. Dominicus Mandić, O.F.M., Chicago / Roma: Institutum Chroatorum Historicum, 1962 ; Đaković (Luka), op.cit.; Džaja (Srećko M.), op.cit., n. 5 p. 150. La comparaison entre Mandić et le manuscrit montre certaines divergences ; mon étude n'est cependant pas le lieu de cette comparaison.

57. Sur cette méthode, voir CADN Sarajevo, vol. 3, 15.IV.1872 (le consul arrive à des résultats différents car il ne disposait pas de tous les chiffres connus aujourd'hui).

58. Đaković (Luka), op.cit., pp. 12-14.

59. ШЕМАТИЗАМ ПРАВОСЛАВНЕ МИТРОПОЛИЈЕ И АРХИДИЈЕЦЕЗЕ ДАБРО-БОСАНСКЕ, Sarajevo : Zemaljska tiskara, 1882. Ce décompte ne comprend pas les éparchies de Zvornik-Tuzla (régions de Sembérie et de Tuzla) et de Zahum-Herzégovine (Herzégovine orientale et occidentale) ; certains villages, en outre, ne sont pas renseignés. Il dénombre 26 proto-presbytères, 238 paroisses, 122 églises, 261 prêtres ; 1805 villages, 46390 foyers et 348585 âmes. Sur ce décompte, consulter JАњАТовЙ (ЂОРБЕ), ПРЕЗИМЕНА СРБА У БОСНИ, Sombor : Prosveta, 1993. 
60. Voir le Jevrejski almanah 5 (1928-29/5690), publié à Vršac.

61. Je n'ai pas encore eu l'occasion de consulter Dündar (Fuat), « Compter, classer, contrôler. Les minorités dans les recensements turcs ", Turcica, 37, 2005. Il est très probable cependant que cet article ne s'intéresse qu'aux périodes ultérieures, en particulier à partir de 1907.

62. "Statistički podatci o Bosni, Hercegovini i jednom kraju Stare Srbije po zvaničnim turskim izvorima ", Glasnik Srpskog učenog društva, 20, 1866, pp. 221-228.

63. Pasco Wassa Efendi, La Bosnie et l'Herzégovine pendant la mission de Cevdet Efendi, Constantinople : Imprimerie du Courrier d'Orient, 1864 (rééd. (utilisée) : Istanbul, ISIS, 1999, pp. $26,65)$. À ce titre, le tableau du recensement de 1851 que l'on trouve chez Giljferding n'est pas complet, puisqu'il ne donne ni juifs, ni bohémiens, et bien qu'il précise le nombre de foyers (ГИљФЕРДИНГ (АЛЕКСАНДАР), «БОСНА ПОЧЕТКОМ 1858. ГОДИНЕ » (La Bosnie au début de 1858), in ГИљФЕРДИНГ (АЛЕКСАНДАР), ПУТОВАњЕ ПО ХЕРЦЕГОВИНИ, БОСНИ И СТАРОЈ СРБИЈИ (Voyage en Herzégovine, Bosnie et Vieille-Serbie), Sarajevo : Veselin Masleša, 1972, pp. 330-332).

64. ХАџИЈАХИЋ (МУХАМЕД), « О МАҢИНСКИМ ЕТНИЧКИМ СКУПИНАМА У БОСНИ И ХЕРЦЕГОВИНИ У XVIII И XIX стОљЕЋУ, ДО ОКУПАЦИЈЕ 1878. », Prilozi Instituta za istoriju, 18, 1981, p. 213-214.

65. Šljivo (Galib), « O Bosni 1864 », in Šljivo (Galib), Bosnien und Herzegovina. Studien und Quellen, Tešanj : Planjax, 2003, pp. 106-107.

66. Karpat (Kemal H.), art.cit., p. 136.

67. Plusieurs versions en sont publiées ou données par les sources consulaires. Les chiffres donnés par le consul français en 1867 semblent les plus exacts et les plus complets, dans la mesure où il les tient du responsable du fisc provincial lui-même. Or, dans cette version, on trouve, en plus des catégories musulmans, catholiques, orthodoxes, juifs, bohémiens (ou zingars), celle des étrangers. On voit donc que les catégories européennes de décompte entrent progressivement dans les méthodes ottomanes.

68. Bernard (Michel), Nations et nationalismes en Europe centrale XIX ${ }^{e}-X X^{e}$ siècle, Paris : Aubier, 1995, pp. 25, 44-49.

69. Thomassen (Bjørn), «The State and the Population Census : The Creation of National Groups in the Austrian Empire ", Jahrbücher für Geschichte und Kultur Südosteuropas, 8, 2006, pp. 21-43.

\section{RÉSUMÉS}

L'objectif de l'article est de détailler les diverses méthodes qui ont prévalu pour dénombrer la population de la Bosnie-Herzégovine à la fin de l'époque ottomane et sous l'administration austro-hongroise. Quoique couramment utilisés dans la presse et les débats politiques, en effet, ces recensements n'ont pour ainsi dire jamais fait l'objet d'une étude critique.

The aim of this paper is to detail the various methods that have prevailed in counting the population of Bosnia-Herzegovina at the end of the Ottoman period and under the AustroHungarian administration. Althoughcommonlyusedin thepressand political debates, thesecensuseshave actually almost neverbeenstudiedcritically. 
INDEX

Index géographique : Bosnie-Herzégovine

Mots-clés : Autriche-Hongrie, Bosnie-Herzégovine, Empire ottoman, recensement de population

Keywords : Austro-Hungary, Bosnia-Herzegovina, Ottoman empire, population census

\section{AUTEUR}

\section{PHILIPPE GELEZ}

Maître de conférences en Études slaves (BCMS), Université Paris Sorbonne Paris IV

philippe.gelez@paris-sorbonne.fr 